\title{
EWB-USA and EPICS: Academic Credit, Community Impact, and Student Learning
}

\author{
William Oakes, P.E. \\ $150^{\text {th }}$ Anniversary Professor, Professor of Engineering Education \\ Director, EPICS Program \\ Purdue University \\ West Lafayette, IN 47907 \\ oakes@purdue.edu \\ Susan Khalifah \\ Director of Student Experience, Civil Engineering \\ Purdue University \\ West Lafayette, IN 47907 \\ skhalif@purdue.edu \\ Claire Sigworth \\ Masters Student, Department of Anthropology \\ Purdue University \\ West Lafayette, IN 47907 \\ csigwort@purdue.edu \\ Paul Fuchs \\ Senior, School of Aeronautics and Astronautics \\ Purdue University \\ West Lafayette, IN 47907 \\ fuchsp@purdue.edu \\ Amanda Lefebvre \\ Junior, Civil Engineering \\ Purdue University \\ West Lafayette, IN 47907 \\ alefebvr@purdue.edu
}

Abstract - EWB-USA engages thousands of students nationally on community-driven projects almost exclusively as an extra-curricular activity. This paper documents how one EWB-USA chapter used the EPICS framework to merge the club into a credit-bearing program that leverages the infrastructure of the university and recognizes the faculty work within their respective teaching loads. The approach preserves student leadership and ownership while enhancing student learning and accountability. Student reflections were analyzed and showed evidence of learning about design as well as broader professional skills including communication, teamwork and project management. Critical and reflective thinking is evident in the class artifacts. Deeper learning including evidence of an awareness of ethnocentrism and positionality and bias were documented in the analysis of the student reflections.

Index Terms - Community-based learning, service-learning, academic credit, reflection 


\section{INTRODUCTION}

In today's global economy, industry needs practicing engineers have to be strong technically and possess the broader set of skills that allow them to lead innovation, work across disciplines, and thrive on multicultural teams. The National Academy of Engineering's (NAE) Engineer of 2020 described the attributes for the globally competitive engineer. ${ }^{1}$ The attributes for graduates include traditional disciplinary fundamentals along with robust communication, teamwork and leadership skills, along with well-developed social and cultural competencies, strong creativity and wideranging transferable skills to address the problems of today and tomorrow, such as the NAE's global "Grand Challenges". 2 The companion report Educating the Engineer of $2020^{3}$ described curricular reforms needed to develop these broader sets of skills. These calls for reform are not new and are similar to those motivating the ABET accreditation guidelines. ${ }^{4}$

Service-learning or community-engaged learning is pedagogy that has shown success in developing the broader skillset needed for today's graduates and has generated a great deal of interest from faculty, students and employers. Students have initiated numerous chapters of Engineers Without Borders USA (200+), Engineers for a Sustainable World (30+), and Engineering World Health (20+). The American Society of Engineering Education (ASEE) has a large division focused on Community Engagement. Community-engaged learning has been shown to have an impact on learning, recruitment, diversity and retention within engineering. Even with these benefits, engineering has been slower to adopt the pedagogy than many other disciplines within the U.S. ${ }^{5}$

Community-engaged learning can also address the reality that many people lack access to the basic human needs of water, sanitation, food sovereignty and education. Appropriate technology can make a significant impact in many of these situations, if applied in a culturally sensitive manner and including cultural mores and norms in the design considerations, from technology selection to development and implementation. Organizations like Engineers Without Borders (EWB-USA) seek to address these needs as they bring students and professionals together. Traditionally, EWB-USA chapters have been outside the traditional curriculum with students doing the design work, culture and travel work in addition to their studies and workload. There are cases where students are given credit, such as at Tulane University, as part of their service-learning hours and at Harvard University, where a credit option is offered. There is no widely accepted model for integrating the work into the curriculum. This paper documents how Purdue University has integrated an EWB-USA chapter into a service-learning curricular structure to preserve the students' leadership, increase student accountability and learning, and leverage the university infrastructure to increase the support and sustainability of the EWB-USA chapter.

\section{EPICS AND EWB-USA HISTORY, COLLABORATION AND OVERVIEW}

\section{EPICS Program}

EPICS was created at Purdue University in 1995 in the School of Electrical and Computer Engineering under the direction of professors Leah Jamieson, Hank Dietz and Edward Coyle. In that first year, 40 students worked with five local community partners to address needs in the local area. $^{6}$ Over the years, EPICS has grown in size and scope engaging both local and global community partners. ${ }^{78}$ In the 2018-19 academic year, over 1200 students were engaged in more 
than 130 projects with 57 community partners from the local community, regional including the Oglala Lakota College and in eight other countries. ${ }^{9}$ The courses is broken up into sections with each section having one or more faculty, staff or industry advisors, one graduate teaching assistant and 10-20 students. It has become explicitly multidisciplinary with an average of about 30 majors participating in a typical year. Students are assessed based on how they apply their disciplinary knowledge and skills to the project work. ${ }^{10}$ EPICS includes students in their first-year to their fourth or final year and the assessments also account for their academic year.

Each department or school within the university determines how the course credit counts in their curriculum. EPICS can count towards graduation in every college and department within the University for at least one course. In some programs, the course credit can be used to fulfill multiple requirements such as a science, society and technology requirement for the university and an entrepreneurship elective for the entrepreneurship certificate. For all of the engineering disciplines, the courses will satisfy at least a technical elective and can be used as capstone for four disciplines. When students elect to use EPICS for their capstone, there is an additional process they have to fulfill that includes oversight from faculty in their home department to insure the capstone requirements are met. The curricular structure is designed to allow students to participate over multiple semesters or even years which supports long-term, reciprocal community partnerships. The long-term student participation allows projects to be developed over multiple semesters or years and allows projects to address complex and compelling needs in the local or global community.

EPICS has been recognized for its curricular innovation by the NAE with the Bernard M. Gordon Prize for Innovation in Engineering and Technology Education, 2004, and as an exemplar of programs "Infusing Real World Experiences into Engineering Education (2012); by NSF's Corporate Foundation Alliance as an Exemplar Program (2002); and by the American Society for Engineering Education with the Chester Carlson Award for Innovation in Engineering Education (ASEE) (1997 and 2012). EPICS was recognized as a signature program of the IEEE Foundation (2013). The success of EPICS motivated the dissemination of the model to more than 50 other universities globally. ${ }^{11}$ Further expansion of the model into high and middle schools created the EPICS K12 program that now have more than 100 schools in the U.S. and over 30 abroad through a partnership with IEEE.

\section{EWB-USA}

EWB-USA is a nonprofit humanitarian organization established in 2002 at the University of Colorado Boulder to support community-driven development programs worldwide through partnerships that design and implement sustainable engineering projects. Since its founding, it has seen explosive growth. EWB-USA currently has more than 14,000 student and professional members in nearly 300 chapters nationally. Each chapter works on programs in the developing world under the guidance and approval of EWB-USA.

Using student chapters as extracurricular activities has allowed EWB-USA to spread rapidly with student interest as students could establish chapters without going through the process to change curriculum. Results of the EWB-USA experience have been widely lauded by industry and within academia. Student leaders in EWB-USA have been recognized with awards including recent recipients of Rhodes scholarships in 2011 and 2013 and Marshall Scholarships in 2012. A team won a prestigious EPA Award for Sustainable Erosion Control in Developing Countries 
Using Industrial By-Products. The National Academy of Engineering inducted the EWB-USA founder, Bernard Amadei, into the Academy for his contributions.

Collaborations and interactions between EWB-USA and the EPICS Program began as early as 2004 in the very early years of EWB-USA when both programs were invited to speak on panels and events that discussed engineering and community engagement. In those years, EWB-USA was an exclusively extra-curricular and global program and EPICS was exclusively a local program with academic credit. Collaborations continued with Bernard Amadei speaking at an EPICSsponsored conference hosted at the National Academy of Engineering in 2006. As both programs evolved they grew to have more in common. EWB-USA included domestic programs in their portfolio and explored ways for students to earn academic credit and EPICS expanded to include global partnerships. The leaders of the programs co-presented workshops at the Annual ABET Symposium and a faculty development workshop in 2014, following the Annual Conference of the ASEE along with other leaders in the engineering community-engagement. It was recognized that both programs shared the common goals of addressing compelling needs of the underserved domestically and internationally while equipping the future leaders in engineering education. Each has strengths and successes that are complementary. Both organizations are committed to engagement that addresses human and community needs through engineering design as well as establishing and valuing long-term partnerships with communities while delivering tangible results with their community partners.

The idea of blending the two approaches was to create a model that would preserve the values of students' learning and community engagement for both programs as well as the positive attributes of the student chapters of EWB-USA. The project began in the fall of 2014 and is continuing at present. The student chapter committed to a new program in 2018 along with the EPICS Program. ${ }^{12}$ As part of this process, the EWB-USA students and the EPICS Program committed to continue the blended model for at least another five years. This paper documents the structure of the model and provides an analysis of student learning demonstrated through written reflections.

\section{Creation of the Course}

While the leadership of EWB-USA and EPICS agreed that an integration would have value, the student chapter was organized and governed by the student leadership who needed to be part of the discussion and implementation. In 2014, the EPICS director approached the student leaders with the proposal of a blended model. Interestingly, a parallel and independent discussion had been going on within the EWB-USA chapter's student leadership team. Some of the officers had experience with EPICS and saw benefits of collaborating to leverage the EPICS structure and support resources. They also saw how the benefits could address some of the challenges in the club including accountability among members and sustained participation over the entire semester. Some on the team had hesitations about potentially relinquishing the student ownership and leadership that is central to the EWB-USA experience. They were also advised by others at the university to be careful of a "take-over" by the EPICS Program. While this last issue is a local and possibly personnel dependent issue, it is shared as an example of how local political and personnel issues are often parts of any new endeavor that have to be acknowledged and addressed. 
The EPICS faculty negotiated with the EWB-USA student leaders to develop a basic framework and philosophy to build the new blended model together and evaluate the success and challenges. The agreement was for a partnership of equals to co-develop the new model whereby EWB-USA students would earn academic credit through the EPICS courses. The chapter had one active program and the first assessment trip had already been made. The results of the first year were presented at the Annual Conference of the ASEE. ${ }^{13}$

\section{ORgANiZATION}

In the spring of 2014, a basic framework for the course was established. The class time was selected as an evening class from 6-8 pm so it did not interfere with other classes. This was the same meeting time that the club had for its meetings so the students knew it would work. It was a change from the traditional EPICS classes that were between 8:30 a.m. and 5:20 p.m. EPICS labs traditionally meet for two hours so this was kept constant. A graduate teaching assistant was assigned to the class along with a faculty member which was also the same pattern as with other EPICS teams. The EPICS faculty advisor also became the EWB-USA chapter advisor.

In the first couple of years, there was an explicit effort to maintain the club structure in addition to the course. Enrollment for the course was restricted to the core leadership and the design team members with other participants operating in the club and meeting in a different location. Students earned their way into the core teams and the class by coming to the club meetings and demonstrating interest and dedication over the previous semester or year. Fifteen students were registered for the Fall Semester of 2015. The number expanded to 19 in the spring of 2016 to accommodate younger students who had shown significant dedication and interest in the design activities. The separation into a class and club created logistical challenges and perceived barriers for students to join. The advantages of the division included having dedicated and reliable students on the core functions that were engaged during the entire semester. Officer duties were changed to accommodate the mix of the club and class and this took significant effort to create authentic work for the club.

In 2017 the club was fully integrated into the class with the consensus of the faculty and students. Keeping the club separate was too resource intensive and the student leaders voted to integrate fully into the class. A typical EPICS section has no more than 25 students and an exception was made for the EWB-USA team necessitating a move to a new classroom. The evening meeting time allows the team to meet in the spaces designed for the first-year engineering courses. A space for new members coming to explore the club/class was created with an officer assigned to oversee the process. All of the members who committed to formal responsibilities enrolled in the class for credit. This change came as a new program was added to the chapter with the five-year commitment being made by both EPICS and the EWB-USA chapter. Currently, student teams include the technical and cultural teams related to the active programs. A separate team is comprised of the leadership team including the president, vice-president, treasurer, etc... This team manages the overall operation and project management aspects of the team. Another team was added to handle the business, fundraising and marketing aspects of the projects. The leadership roles that blend EWB-USA and EPICS positions are shown in Table I. 
TABLE I: STUDENT Roles

President is the overall leader of the club and responsible for the management and leadership of the club

Vice President assists in leading the overall club, manages the meetings for the club and recruitment and education of new members

Treasurer is responsible for the management of the club's finances. This is a recognized university position and is required by the university to be a recognized student organization

Project Manager is responsible for communication between the team and the project partners and is responsible for organizing design reviews

Head of Fundraising works with the treasurer and their team to write grants for travel and project funds

Media Head works closely with the head of fundraising and treasurer to manage and update the website, social media and promotional projects for the club

New Member Design team leader integrates new, non-EPICS members of EWB-USA into the curriculum by teaching them the design process and presenting them with a smaller challenge to complete during the semester

Design team leader is responsible for overseeing the design teams and completing semester objectives directed towards the design project(s)

The full integration of the chapter into the credit bearing was enabled in part by the fact that Purdue University has a block tuition model so students do not pay more for one or two additional credits. EPICS can be taken for either one or two credits per semester and each student makes the decision based on their expected workload and time available. Students are allowed to register for EPICS courses for as many semesters as they desire. If students were willing to make the commitment of the time for one or two credits, then they were given a role on one of the teams.

The creation of the leadership and business teams within the course required an expansion of the thinking of the traditional design roles. EPICS is a design course and the work of the leadership team is directly related to the success of the overall project. That is consistent with other EPICS divisions where students take on different roles within the overall project and partnership depending on the needs of the partnership and their own expertise and area of study. The work in project management, community partnership building, fundraising and other activities are approached with the lens of design. The fundraising, for example, is evaluated on the systematic development of a plan, its execution and the learning that follows. Students are evaluated on how well it has been developed and what they learn rather than the amount of money raised.

All EPICS students develop a plan with their faculty advisor for each semester and are assessed based on those expectations for that semester. The EWB-USA section is not different in that regard 
but has a more formal structure with more officer positions. The assessment process allows students to participate with diverse backgrounds and skills and to take on different roles each semester. Students are also placed on the teams based on their expertise, interest and what kind of credit the EPICS class counts for within their respective department. For example, a junior or senior in Civil Engineering taking EPICS for a technical elective credit would not be placed on the business and marketing team but directed to a technical team. This requires faculty to be involved in the team assignments and semester expectations.

The current lab structure, still 6-8 pm, has the entire club meeting as a whole for the first twenty minutes to go over the reports from the teams using a Progress, Issues and Goals format for the past and upcoming weeks. This is a common reporting format used across all of the EPICS divisions and each EWB-USA sub-team reports out so all of the members see what is going on across the organization. During this time, new members who are not required to join EPICS are able to get an idea of what fully committing to EWB-EPICS entails. This interaction between new members and EWB-EPICS members is essential to the growth and education of the growing team.

After the review, the teams meet for the remainder of the lab time and the advisors and teaching assistants consult with the teams. New members are directed to their own area where members of the leadership team dedicated to the new members facilitate programs that engage the students in design tasks related to the programs or fundraising activities that are part of the overall club. The intent is to give the new members a taste of what the EPICS-EWB experience is like and for them to demonstrate that they have a commitment to EWB-USA and can be a contributor to the organization.

Most students are required to participate in the new members' process for at least one semester but exceptions were agreed upon. One is for students who have a proven record for similar experiences, such as being on another EPICS team for multiple semesters with a local or global partner. The other are advanced students, often seniors, with specific skills that would significantly aid the project teams. Exceptions are made on a case-by-case basis when students come with prior experience or needed expertise. They are recommended by the student leaders and approved by an advisor.

EPICS students are expected to meet as a team outside of lab during the week to further their work and the EWB-USA team does the same. Along with the project work, Professional Development Hours ( 5 for one credit and 10 for two credit students) are required for all EPICS students and significant freedom is given to students to select how to meet these hours. Many of the EWB-USA initiatives can be counted as PDH's, including attending an EWB-USA conference. Students who take the course for multiple semesters participate in different tracks and options for their PDH's and take on different roles within the team to continue their development. Generally this involves more responsibility but in semesters where their load is heavy outside of EWB-USA they may choose to cut back. As mentioned earlier, the expectations for each student is planned at the beginning of each semester with the advisor and teaching assistants.

Enrollment in the course has grown to over 40 members with a varying amount of new club members that are learning about EWB-USA, EPICS. There continue to be challenges but the belief amongst the faculty and student leaders is that the credit-bearing model has enhanced the learning and the performance of the EWB-USA team. 


\begin{abstract}
AdVISORS
When the blending first started, the Purdue EWB-USA team had a travel mentor from the Indianapolis professional chapter. This person was the Responsible Engineer in Charge (REIC an official EWB-USA position for each program) for the active program. The EPICS faculty member served as the local mentor and chapter advisor and was responsible for the assessment and grading and mentoring the team on a weekly basis. The two worked together to mentor the students and came to agreements on who was responsible for which aspects of the students. The REIC travelled with the team and the EPICS faculty member handled the course grading and logistics and preparation for the trip. In the second year, a change in faculty advisor was needed for a year due and another advisor stepped in with the same the REIC. A professional EWB-USA member joined the School of Civil Engineering and joined the advising team. Currently, two EPICS advisors work with the local team and one travels with the team along with the REIC. A new REIC was added for the new program who travels along with one of the advisors. The change in advisors has shown that the model is not dependent on a particular faculty member or professional mentor.
\end{abstract}

\title{
Travel And Study Abroad
}

To complement the EWB-USA and EPICS class partnership and fulfill the needs for travel, a formalized study abroad program for credit has been established. This allows students to travel as needed for EWB-USA projects while also receiving credit for the work they are performing while engaged with the community. The credit bearing model also facilitates student reflections to to assess their learning before, on and after the trip. The study abroad integration allows the EWB-USA team to utilize the staff who assist in the planning and execution of study abroad trips support the study abroad programs. Financially, students are not charged tuition for any study abroad but rather a fee. They are given access to scholarships that can offset these fees and reduce the cost of the trips. The scholarship are need-based and given based on family reported income. The decision to move to a formal study abroad model was made, like the other decisions, as a collaborative discussion with faculty and students and arrived as a consensus.

The linkage with the study abroad did not impact the number of days that were planned for each trip. The number of credits for each trip is determined after the length of the trip is finalized and in consultation with the Global Engineering Program staff. Pre-trip activities include a set of required sessions on the culture and expectations for the travel team. These are not significantly different than what the club would have planned but it holds students accountable for the activities. During the trip each student keeps a written journal and the team holds reflection sessions most days in the trip. After the trip is over the team writes the trip report, each student writes a summative reflection and the team presents their experience and the results to the rest of the EWBEPICS team.

\section{ASSESSMENT}

The assessment process for the EWB-USA section follows the same principles, philosophy and methods as the other EPICS sections and is guided by two core values. First, it seeks to provide an educational experience that will prepare students for professional practice, by modeling the 
practices used in industry and professional organizations. Students set goals at the start of their semester based on their role, status of their project and the capabilities of their team. They are assessed similar to a performance evaluation on their attainment of these goals. Since it is an educational program, there are learning goals as well as project accomplishments but the philosophy of a performance-like assessment remains.

The second philosophical value is that we seek to meet compelling human, environmental or community needs. The assessment processes are integrated into the design work to encourage progress on the projects while explicitly considering the partnership, cultural dimensions, communication and teamwork. The dimensions that are assessed include accomplishments, design process, critical and reflective thinking, communication and teamwork.

The assessment process evaluates authentic project artifacts (papers, reports, notebooks, blogs, reflections, etc.) that can be used to demonstrate student learning and achievement. The EWBUSA reporting structure is rich with team-based reports and requirements that are used in place of the traditional EPICS design documentation. Wherever an EWB-USA report or requirement can be used to fulfill an EPICS assessment it is. Students also keep an electronic notebook in Microsoft OneNote where they record meeting minutes, notes from their work during the week and individual weekly reflections that are graded. Students have commented in their notebooks how the notebooks and reflections that add work to their normal EWB-USA activities. They have also written about their usefulness in their reflections. The professional mentors have helped reinforce that the practices of notebooks and reflections are both beneficial practices for professionals in our fields.

Students are evaluated informally in week four of the semester to ensure that they are documenting enough. A more comprehensive formative grading occurs at the mid semester mark in week 8. With each grading, the graduate teaching assistants organize and review the student materials and work with the faculty advisor to determine the grades. The process and grading rubrics can be found on the EPICS website. ${ }^{14}$ The intent of this formative grading is to have a conversation with the student about their progress, learning and the documentation they are producing to make a proper assessment. Often, students are not documenting sufficiently to properly represent their accomplishments and learning. The formative assessment allows students and faculty to calibrate their expectations. Peer evaluations facilitate the evaluation of teamwork, help to delineate individual contributions, and are done at the middle and end of the semester.

The same process is done in the summative evaluation at the end of the semester. The instructional team collects and assesses the artifacts, uses the comments and grade given to the student at the mid-semester and arrives at a grade for each student.

\section{STUdent REFLECTIONS AND EVALUATIONS}

The data to assess the impact of the blended model with the EPICS curricular structure and the EWB-USA chapter includes summative course evaluations. In the first year of the transition into EPICS, the students rated the course and instructor with a 4.9/5.0 $0^{15}$ and the evaluations have continued to be between 4.5 and 4.9 out of 5.0. Evidence of the success also comes from the continuation of the program and the student comments. In 2017 the student leadership team evaluated the collaboration and chose to fully integrate the club into the EPICS curricular structure. Adopting the new program connected the EPICS Program and Purdue's EWB-USA chapter for at least five more years. The success has also been shown in enrollment demand for the EWB section 
of EPICS. Starting with 14 active members, the 2020 enrollment will exceed 50 and continues to grow.

Other evidence of the success can be found in the words of the student leaders. The chapter president in the first year of the collaboration was asked about the experience by another chapter and if he recommended looking at it for their own institution. His email reply was that he saw many benefits and that he would recommend any university chapter adopting a similar model.

The project manager of the first EWB-EPICS class had been in EPICS for two years. Her perspective was:

Trying to combine the EPICS and EWB-USA clubs required a lot of planning and critical decisions, and although it was and still is a challenging process I believe it is the best thing to have happened to our EWB-USA team. Prior to the EWB-USA-EPICS Team, our EWBUSA has to organize ourselves into a cohesive, yet delegating team to solve a complex engineering problem. So much time of the club was spent exhausting our resources and trying to solve the problem, through whatever unorganized means necessary. Many times the responsibility of deliverables fell onto one team member because there was not a clear set of repercussions for incomplete work aside from a delay in project progress. Having a more organized, class-setting where each team member is held accountable for their work has tremendously improved the efficiency and progress of our project. Having faculty support and guidance from the EPICS structure has aided our design team significantly as well... Trying to organize the logistics of the combined EWB-USA and EPICS team has proved to be an excited and challenging process that I have enjoyed being a part of ${ }^{16}$

The current president who earned the EWB-USA Leadership Award in 2019 was asked to comment for this paper and her reaction was

Integrating EWB Purdue with the EPICS program creates more accountability between chapter members. Each person has a role to play in the success of a project. The requirement for students to enroll in the EPICS program to be involved in the design teams separates who is passionate about the project from those who aren't. In addition, it creates more incentive for students to get things done because of repercussions reflected in a grade.

\section{STUdent Learning}

Reflection is a requirement of the EPICS courses, both as a weekly activity and a summative learning activity. The reflections were analyzed for the Fall of 2018 semester, , to evaluate how and what students were learning from their EPICS-EWB experience. All of the student reflections were analyzed by a graduate student from Anthropology with training in qualitative data analysis. The qualitative analysis was done under the guidelines of an exempt IRB protocol for academic artifacts in the EPICS Program. The main themes that emerged from this analysis include:

\section{Human-centered design}

The human-centered design process is integral to the EPICS experience, as it is the design method that melds both the human and the technical. Students acknowledged the benefits of a humancentered design process in relation to their engineering projects, citing not only the value of 
learning how to work on a collaborative design team, but also how to create design projects that are valuable for their intended users. One student spoke specifically about this, stating that:

"The iterative process combined with the user center focus allows you to design something functional and then improve its functionality."

This user- or human-centered focus has encouraged students to not only keep the communities they are working with in mind throughout each design stage, but to really understand the lived experiences of their users and find ways to make design outcomes effective in different contexts. This melding of different types of knowledge -- about culture, environment, and technology -- is something students have also explicitly recognized as important:

"When I began this semester, I thought that Human-Centered Design was such a cool way of approaching engineering problems. Now, I feel like I am actually learning how to go about achieving it by asking the right questions, and analyzing them the right way, to combine our knowledge of the community and their environment and our technical knowledge. It is so much deeper than originally anticipated, and I feel like I am just brushing the surface in all the ways you can go about trying to cultivate understanding of your client..."

Some of these different modes of understanding come from the chapter's interactions with those beyond the discipline of engineering, such as anthropology and communication. Each semester, guest speakers are brought in to discuss topics relevant to the projects underway and to international development and community engagement more broadly. These opportunities to engage with related discussions from other disciplines had broadened students understandings of what community engagement can look like, and have encouraged students to think more about the larger implications of their work:

"Already, in my short time with EWB, I have learned so much about the cultures and customs of our community partners...listening to guest speakers regarding the anthropological implications of the project has piqued my curiosity--the ability to understand our community partners on a cultural level is incredibly important."

Not only are students recognizing sociocultural understandings as critical components of their work, but they are also seeing how the human context and value-systems can play a role in shaping the outcomes of a design project:

"The idea that community partner cultures and desires push our innovation is not only a phenomenon that fascinates me -- I now see it as a necessity."

In addition to learning about the human-centered design process and the impact cultural understanding can have on design and engineering projects, students also reflected on their own positionality within these processes and grappled with questions about why they were so passionate about the project and how they were perceiving it. These two quotes nicely sum up this learning:

"I definitely had the mindset that we were coming to save and help the members of this community. Now, I understand that we have so much to learn from them about their ways of life, and that their culture and ways of living are just as worthy of respect and understanding as our own." 
"Personally, I have found that I love engaging in international development work. But, initially, I loved it for the wrong reasons--I felt compelled to get involved because I arrogantly thought the world needed me to help. However, my convictions have now changed."

\section{Professional Preparedness}

In order to receive a grade in the EPICS program, students are evaluated based on five main criteria: (1) Accomplishing Project Goals, (2) Utilizing the Design Process, (3) Reflective/Critical Thought, (4) Teamwork/Leadership, and (5) Communication. These categories encompass many of the learning objectives of the EPICS curriculum, and include other implicit learning objectives, such as project and time management, collaboration, documentation, and budgeting. Students draw on many of these themes in their reflections, making their learning explicit, and talking about the ways in which their EPICS-EWB experiences have enhanced their learning and their growth in these areas.

\section{Communication}

The first theme, one explicitly stated on the EPICS rubric, is communication. In their reflections, students acknowledge the benefits of learning to communicate both within a team and to external reviewers of their projects. Additionally, they recognize the importance of communication to a diverse range of team members and stakeholders throughout the design process, as a vital part of collaborative, human-centered learning:

"I have developed communication skills and collaboration techniques that I never would have thought of before becoming an engineering student."

"Working on a human-centered design, engineering project through Engineers Without Borders has allowed me to enhance communication and teamwork skills...The variety in my teams also allows me to grow my communication abilities...The intricacy of this project forces us relay research and findings to one another so we can all accomplish our goals as a team, not as individuals."

As mentioned above, twice a semester, students present their projects to a panel of reviewers. This not only gives them the opportunity to gather feedback about their project and their progress, but it also gives them the chance to practice talking about their work to other audiences and in a presentation setting. Students have recognized the value of these presentations as useful tools for improving their communication skills:

"After giving the presentations, I felt a lot more confident about my public speaking skills. It is so essential that I improve my communication skills and this design review was a big step towards improving it."

\section{Teamwork and Collaboration}

In addition to communication, teamwork is another criteria on which students are evaluated. The collaborative and dynamic nature of EPICS-EWB design projects allows students to practice 
working effectively on a team in tangible ways. Students have recognized this direct link between the human-centered design process and the necessity for teamwork, stating:

"I do not think I would be nearly as proficient in working on a team if it were not for the human-centered design that we are developing in Engineers Without Borders."

There were some students who came into the EPICS-EWB classroom, nervous that they would not enjoy the experience based on preconceived notions and past experiences of teamwork, who found that working on collaborative design teams is an enjoyable process:

"I have always said that I hated teamwork, which made the idea of becoming an engineer and working in a team the rest of my life a little bit scary. However, through EWB I have learned that I greatly enjoying working in a team environment when surrounded by capable, motivated people."

Students have also recognized that this type of collaborative, project-based work can better prepare them for the workforce:

"I am going to move on to a job or on to graduate school and I will have a boss.

These subgroups and this team are a training ground for the challenges I will face in the workforce."

Some students even mentioned that, when beginning their job searches, they would start looking for firms that work through a similar collaborative design process, because they see its value:

"I would place emphasis in a job search on finding a place with a fast paced collaborative environment where prototyping and decisions can be made, seen through, then adjusted per feedback just like the EPICs process"

In addition to working alongside other engineers, students are working towards broadening their disciplinary scope by bringing in those from other disciplines, as mentioned in the above section, such as anthropology. Students see the value in having multidisciplinary teams and incorporating other disciplinary knowledge:

"[Community engagement] has been a big focus within our team, and we have sought to bring in anthropologists. Input from non-engineers has been extremely valuable to our team."

\section{Ethics}

In thinking through their projects, students also acknowledge and grapple with ethical decision making regarding design decisions, and recognize that ethics can be a messy process:

"As engineers, we often look at the ethical approach as doing the greatest good the many while cutting the least harm. This is seemingly obvious, but could cause a great harm to the few, and this realization has changed my perspective on ethics as a whole. I think moving forward, I would have to look at ethical dilemmas in multiple ethical viewpoints."

Students use their reflections as a space to talk about the consequences their designs may have within the communities they work with, and on their stakeholders: 
"This semester has taught me a lot about conscious, ethical decision making and the importance of deeply understanding the people we work with, their culture, and their frame of mind, and not just your own, when making design decisions and thinking deeply about the consequences and impact your engineered solutions will have on your clients and stakeholders."

\section{Organization and Time Management}

Students also reflected on the importance of being organized, and properly planning both one's individual schedule, but also the team's design schedule. While time management is not an explicit learning outcome as designated on the EPICS rubric, it is implicit in the grading criteria of Accomplishing Project Goals. Breaking large goals down into manageable chunks is a necessary life skill for both personal and professional life, which is something students have recognized in their reflections:

"EWB requires so many little parts along with paperwork that has to be documented for Purdue. Breaking this down into individual sections helps ensure we meet deadlines and not get overwhelmed in the process. This is a great lesson in project planning/management that I can transition into other areas of my life especially when I graduate and work in the real world."

"I have always found it hard to find the balance between over planning and underplanning...I work very well when I have an end goal to work towards, but it is setting challenging yet attainable goals that has been the issue...I have a couple of larger tasks on the timeline that should probably be broken into more manageable parts."

"So, I recognize that time management, especially when asked to perform various tasks, is important for not only yourself in the moment, but for yourself 1 hour from now, yourself 2 hours from now, yourself 1 day from now, etc."

\section{Budgeting and Project Finances}

Participating in a service learning program in international development has also given students insight into how project funding happens. In their reflections, they have talked about how this topic is rarely taught in the engineering classroom, and is actually best learned outside of a traditional classroom setting. The experience of working on a "real world" project in the safety of a learning environment, and under the supervision of experienced advisors, gives students more hands-on engagement with the nuances of design work, as recognized by this student:

"I also had to learn how to manage funds and began to understand the total cost requirements for an international trip such as this. These are all skills which will be important to my success in a future career, but are not always taught in undergraduate engineering programs. Being able to participate in project implementation and monitoring through EPICS has given me the opportunity to participate in a real world project and discover the nuances of engineering design that cannot be taught in the classroom." 
Students in EPICS-EWB take a long-term look at their project finances, recognizing that instead of thinking of their budget in increments, it has to be looked at in the same timeframe as the project itself. Students use their reflections to consider ways in which different organizational and funding strategies can be implemented or changed within the chapter to promote more efficient work flow and to better support the project monetarily:

"Due to our 5-year commitment and the community's necessary 5\% contribution, it may be more important than we think for us to introduce the discussion of finances in our correspondence with them. Thus, having a financial outline as a team is incredibly important....This means that similar to our 5-year plan for the project, we should have a 5-year financial plan. Thus far in the history of the chapter, we have only taken finances as they come, not really looking at future repercussions--simply trying to garner as much money as possible per semester. This outlook needs to be changed a little bit. I feel that the chapter would benefit from having set semester goals on how much money we want to earn. Further, we should also create a more detailed semester budget that includes how much money we want to be spending on events."

\section{Documentation}

When students come into the EPICS program, they often complain about documentation. Students see it as an assignment -- a type of busy work -- that is not integral in the design process. However, by the end of the semester, students often reflect on its importance and value for the current team as well as future ones:

"Although before this week I had the assumption that documentation is unnecessary for the design process, I now understand that it is definitely something that is a useful tool for establishing what is known, coming up with new details, finding new possibilities, and establishing what is most important."

In addition to seeing the value of documentation for the design process more broadly, students make direct connections between documentation and their professional futures. They begin to see the benefits of documentation as multiplex -- useful for themselves to look back on their work, useful for others to see their progress and replicate their design process, and useful for other stakeholders as well, such as clients, users, and the broader public:

"A real job would involve a lot of paperwork and everything I do would have to be documented. Documentation is really important so that other people, or some person who is going to take over my task has an idea of what I did. I will make sure to organize and document everything properly. Documentation is a big part of the design process."

"When I become a professional in my career, I will always try to stay organized and try to document everything I do in terms of conferences, meetings, etc. for future reference. Not only could it serve as a reference for me, but it could also serve as a reference for other employees, customers, or just the public (if they want to know about the progress I have made in a project)." 
In addition to these learning objectives above, there is also evidence that students are expanding their knowledge beyond the core course outcomes into deeper understandings and implications of design and engineering work, especially in cross-cultural and international contexts. Some of these broader themes include ethnocentrism, the evaluation of other cultures based on one's own culture and perceptions, as well as the varied and multiple ways in which positionality and bias are a part of the design process.

\section{Ethnocentrism}

Ethnocentrism is a concept stemming from anthropology and sociology, and is a comparative mode of cultural understanding which frames other people's cultures in light of one's own. As evidenced in their reflections, students are thinking about this concept within relation to their own work through international development, both through the actual implications of development work, and even the language of development itself. Some students even take the initiative to learn about these concepts upon themselves, and encourage their fellow team members, especially those preparing for travel, to collectively read different pieces related to cross-cultural understanding, culture shock, and cultural relativism. One student reflected on a piece they had read, to talk about how it can be so easy to oversimplify and reduce other people's problems, especially when removed from the context via culture or geography:

"Obviously, when we read about a kid who thinks he can come to America and solve our gun legislation laws, we think he is diluted and does not understand the problem. Yet, this must be how many of the communities we work with feel when we go there to introduce a new piece of technology. We seem to think that implementing a piece of technology will resolve a plethora of issues and is feasible to complete within five years."

While this student recognizes the value of new technologies, they also acknowledge the grand challenges of today are not solved by technology alone -- that these problems are more complicated and more intricate than an outsider may believe.

In addition to thinking of the actual process of international development as more complicated than a one-size-fits-all solution, students are also wrestling with the concept of language, and are considering how the way in which we talk about development shapes the way we perceive it and the way we go about doing it:

"Dirty streets, thatched roofs, starving people, and limited access to sanitationimages of these sort are painted in our minds when we hear the phrase "third-world country." What we don't realize is that this "first, second, third" classification refers to hegemonic power hierarchies during the Cold War. This term is archaic, yet, it is something we still hear in current jargon when discussing global phenomena. Through working in EWB Purdue, I have realized that rhetoric such as this is extremely pervasive and limiting in the realm of international development, as it works from an ethnocentric viewpoint. Though we may not realize, usage of such phrases creates an implicit attitude in which countries not considered to be "third-world" evoke superiority."

This student is acknowledging the power structures at play within the design process, and realizing that the language we use to talk about other countries only enforces hegemonic and ethnocentric practices. This learning goes beyond the scope of the EPICS learning objectives, and showcases 
how students are engaging with very difficult and complicated, but necessary, questions about their own work, and their own existence within the world more broadly.

\section{Positionality and Bias}

Students are also looking at their situatedness within the context of these projects, and how their viewpoints and their own socioculturally learned understandings of the world relate back to how they work through the design process. Similarly to the theme of ethnocentrism, students are recognizing how their positionality and their own worldviews can change outcomes of design, and can change the way they interact with community members and project partners. One student in particular voiced it this way:

"As engineering students in a wealthier country with more technical training, I think students in our program tend to feel superior to some of the community members they are helping. It is important to keep this mindset in check and make sure it does not come out during our interactions with community partners. We also need to go beyond executing social cues that demonstrate respect, and actually make sure that we all do feel respect for the people we are working with."

Students are also recognizing how biases can play a role in data collection and data analysis. Currently, a portion of students are reviewing qualitative data from a recent assessment trip, analyzing interview data to address user needs and desires. Those working on this initiative are recognizing how their biases, as well as translator biases from the in-country interviews, can shape the way in which data is understood and used. It is impossible to remove bias from the process of gathering and understanding data, and students have learned not to try to avoid bias, but to recognize it for what it is, and acknowledge how it may have skewed the results:

"The reading that I completed this week on the bias introduced by translators, caused me to reflect on all of the potential sources of bias throughout the data collection and analysis process. My first instinct was to try to control all of these potential sources of bias, eliminating as many of them as possible. I later came to the realization that the better option is to accept that many potential forms of bias are present, and to keep these in mind when completing the analysis of this data. There are some things that we can do to cut down on bias, however it is always going to be present. There is bias in the way that questions are asked, the ways that questions are answered, the way the translation is done, the way answers are recorded, and the way these written notes are interpreted and utilized."

"Also this week I realized how difficult it is to draw concrete conclusions from interview data. Multiple biases were introduced because sometimes we are unsure of how they interpret the question, and some household did not have a response to the questions. An important skill to learn from this is recognizing limits that your data has, and recognizing biases that may be present. This is applicable to real industry because there are weaknesses in every set of data and recognizing them is important to be able to make any conclusions."

Overall, an analysis of student reflections have highlighted and made explicit the deep learning that this integrated curricular and extra-curricular experience can provide students. This 
educational experience gives students a well-rounded approach to learning about engineering, international development, and the multidisciplinary, collaborative design process. The structure of a curriculum mixed with the passion and drive students often bring to extra-curricular activities enhances this level of learning, and provides students with the opportunities to critically think about their work and their futures in an academically-supported environment.

\section{DISCUSSION}

EPICS and EWB-USA share many common goals and overall philosophies. Both organizations share goals related to student learning and community impact through long-term partnerships and technology-based, culturally appropriate designs. The integration of the EPICS framework with an EWB-USA chapter was shown to be successful although full of challenges.

Student buy-in during the initial offering gave way to a cohort who wanted into the class but did not want to participate in the curricular activities, including keeping notebooks and weekly reflections, and chose to audit the course. The model where students were participating but not taking it for credit was short-lived and eliminated except in extraordinary circumstances. There continues to be a tension with students asking to participate without credit. It is noteworthy that the student leadership has become very strong asserting that if students can't commit to a credit, they should not be assigned a role on the team. This is in part due to the success of the collaboration and also that they have not known any different model as they started with EWB as part of the credit-bearing model.

A tension from the beginning is what to do with the "club" aspects of the chapter. Initially the students wanted to maintain the feel of the club and have a space where only the club activities took place. The overhead of the club and class led to the full integration into the class with club attributes. The Purdue chapter of EWB-USA is still a registered student organization and maintains the officer positions within the university. Funding comes into the student organization accounts as well as the EWB-EPICS account. This allows the chapter to take advantage of more funding and training opportunities within the university. It has also created problems within the university systems that are used to dealing with academic departments and student organizations. They are not set up to handle something that is both. For example, funds cannot be transferred between the EPICS Program EWB accounts and the student organization EWB accounts.

Callouts are held like other student organization but students are invited to explore the class. The class structure is designed to give all members an overview of the chapter activities and new members who are not registered for the class and assigned roles on the design or management teams are engaged in activities related to the design or fundraising and marketing activities related to the chapter.

The integration has allowed EWB-USA at Purdue to take advantage of the EPICS Program's infrastructure and curriculum. The chapter advisors are given teaching credit like other EPICS instructors. Student earn credit through the EPICS course numbers that are already accepted in all of the engineering disciplines and throughout the university.

Even with the success, there remains a question of how students who would like to be part of a club can participate. There is a way for new members to be just club members but not so for those who have been part of the team. This is especially compelling for seniors or students in their final semester when they are interviewing and preparing to leave. Other students might have a heavy semester and want to scale back. There is an active discussion about what such a role would 
International Journal for Service Learning in Engineering, Humanitarian Engineering and Social Entrepreneurship Vol. 14, No. 3, Special Issue, pp. 29-48, Fall 2019

ISSN 1555-9033

look like but no consensus. There is agreement that it would not involve a formal role on a design team as those require more of a commitment.

Travel logistics have been integrated into the university's study abroad program which leverages the resources to support these programs. It has meant that EWB-USA activities are counted within the College of Engineering's activities and are highlighted among the programs in the college. Students at Purdue are not charged tuition for student abroad but only fees and have access to scholarships that can reduce the overall cost of travel.

The academic scaffolding gives students motivation to stay fully engaged throughout the semester, which was a challenge when the merger was originally considered. Students make a commitment for one or two credits for the semester. The scaffolding also requires students to document and reflect on their work. The analysis of the work shows many aspects of their learning.

The student learning that corresponds to design and the broader professional skills is consistent with analyses across EPICS teams. It is particularly interesting how the users and partners are taken into consideration across the student reflection. Students have discussions and explore the aspects of the cultural differences. Deeper learning surfaces in the reflections when the students reflect on ethnocentricity and positionality and bias. Learning activities and speakers are scheduled during each semester and data from one semester informs the schedule for the next. While they do not name these themes, they are evident in the reflections as the students consider the cultural aspects of the work they are undertaking. This kind of learning may be happening in many other EWB-USA chapters but the academic scaffolding draws it out and allows students to critically reflect on the elements.

Opportunities for the EPICS/EWB-USA credits to count as more, including capstone projects are under discussion. The model works at Purdue University and aspects could be disseminated to other institutions. More than 50 institutions have adopted EPICS into their curriculum in some manner. Each institution is unique in their students, faculty, curriculum, attributes and challenges. Each EWB-USA chapter is different with their students, faculty and community partners. The model shared within this paper is clearly transferrable in a similar manner that the EPICS model is across a broad spectrum of institutions. Like the variation of EPICS adoptions, it would be customized to each local context.

\section{REFERENCES}

\footnotetext{
${ }^{1}$ National Academy of Engineering (NAE) The engineer of 2020: Visions of engineering in the new century. (Washington, DC: The National Academies Press, 2004).

${ }^{2}$ National Academy of Engineering (NAE), " Grand challenges for engineering." National Academy of Engineering, Accessed November 22, 2019. http://www.engineeringchallenges.org/cms/challenges.aspx

${ }^{3}$ National Academy of Engineering (NAE) Educating the Engineer of 2020 (Washington, DC: The National Academies Press, 2005)

${ }^{4}$ ABET, "Engineering Criteria 2002-2003”, ABET, Accessed May 2002, http://www.abet.org/criteria.html

${ }^{5}$ Edward Zlotkowski and Donna Duffy, "Two decades of community-based learning”, New Directions for Teaching and Learning, Vol. 2010, Issue 123, (2010), pp. 33-43

${ }^{6}$ Edward J Coyle, Leah H. Jamieson, Leah H., William C. Oakes, "EPICS: Engineering Projects in Community Service”, International Journal of Engineering Education Vol. 21, No. 1, Feb. 2005, pp. 139-150.

${ }^{7}$ Carla B Zoltowski, and William C. Oakes, "Learning by Doing: Reflections of the EPICS Program", Special Issue: University Engineering Programs That Impact Communities: Critical Analyses and Reflection, International Journal for Service-Learning in Engineering, 2014, pp. 1-32.
} 
International Journal for Service Learning in Engineering, Humanitarian Engineering and Social Entrepreneurship Vol. 14, No. 3, Special Issue, pp. 29-48, Fall 2019

ISSN 1555-9033

${ }^{8}$ William Oakes, Andrew Pierce, Nusaybah Abu-Mulaweh, "Engagement in Practice: Scaling Community-based Design Experiences", Proceedings of the 2018 ASEE Annual Conference, Salt Lake City, UT, June 2018

${ }^{9}$ William C. Oakes, Carla B. Zoltowski, and James Huff, "Engineering Service-Learning: A Model for Preparing Students for Engineering Practice While Meeting Needs of the Underserved", Journal of Engineering Education Transformations, Volume XXVII, No. 4, July-2014, pp. 46-60

10 Antonette Cummings, James Huff, William Oakes and Carla Zoltowski, "An Assessment Approach to ProjectBased Service-Learning”, Proceedings of the 2013 ASEE Annual Conference, Atlanta, GA, June 2013

${ }^{11}$ Oakes, "Engineering Service-Learning: A Model for Preparing Students for Engineering Practice While Meeting Needs of the Underserved"

${ }^{12}$ William C. Oakes, Carla B. Zoltowski, Katherine Schmotzer, Ana Paula Valenca, "Integration of Curricular and Extra-Curricular Learning Through Service," Proceedings of the 2015 ASEE Annual Conference, Seattle, WA, June 2015

${ }^{13}$ Oakes, "Integration of Curricular and Extra-Curricular Learning Through Service,"

${ }^{14}$ EPICS Program, Purdue University, "Grading Summary”, Accessed November 26, 2019, https://engineering.purdue.edu/EPICS/teams/team-documents/Grading

${ }^{15}$ Oakes, "Integration of Curricular and Extra-Curricular Learning Through Service"

${ }^{16}$ Oakes, "Integration of Curricular and Extra-Curricular Learning Through Service" 